\title{
Local Approximate Symmetry of Birkhoff-James Orthogonality in Normed Linear Spaces
}

\author{
Jacek Chmieliński, Divya Khurana, and Debmalya Sain
}

\begin{abstract}
Two different notions of approximate Birkhoff-James orthogonality in normed linear spaces have been introduced by Dragomir and Chmieliński. In the present paper we consider a global and a local approximate symmetry of the Birkhoff-James orthogonality related to each of the two definitions. We prove that the considered orthogonality is approximately symmetric in the sense of Dragomir in all finite-dimensional Banach spaces. For the other case, we prove that for finite-dimensional polyhedral Banach spaces, the approximate symmetry of the orthogonality is equivalent to some newly introduced geometric property. Our investigations complement and extend the scope of some recent results on a global approximate symmetry of the Birkhoff--James orthogonality.
\end{abstract}

Mathematics Subject Classification. Primary 46B20; Secondary 51F20, 52B15, 47L05.

Keywords. Birkhoff-James orthogonality, approximate Birkhoff-James orthogonality, C-approximate symmetry, D-approximate symmetry.

\section{Introduction}

The Birkhoff--James orthogonality is the most natural and well studied notion of orthogonality in normed linear spaces. In general, the Birkhoff-James orthogonality is not symmetric. Chmieliński and Wójcik [5] introduced a notion of approximate symmetry of the Birkhoff-James orthogonality in normed linear spaces. It should be noted that the authors of [5] considered this notion in the global sense, the meaning of which will be clear once we present the 
relevant definition in this section. In this article, our motivation is to consider the corresponding local version of the aforesaid concept. We also study the local version of another standard notion of an approximate Birkhoff-James orthogonality considered in [6]. The advantage of considering the local version is illustrated by obtaining some useful conclusions in the global case, separately for finite-dimensional polyhedral Banach spaces and smooth Banach spaces.

Let us first establish the notations and the terminologies to be used in the present article. Throughout the text, we use the symbols $X, Y$ to denote real normed linear spaces. Given any two elements $x, y \in X$, let $\overline{x y}=\operatorname{conv}\{x, y\}=$ $\{(1-t) x+t y: t \in[0,1]\}$ denote the closed line segment joining $x$ and $y$. By $B_{X}=\{x \in X:\|x\| \leq 1\}$ and $S_{X}=\{x \in X:\|x\|=1\}$ we denote the unit ball and the unit sphere of $X$, respectively, and $B(x, \delta)$ denotes the open unit ball in $X$ centered at $x$ and with the radius $\delta>0$. The collection of all extreme points of $B_{X}$ will be denoted as Ext $B_{X}$.

Let $X^{*}$ denote the dual space of $X$. Given $0 \neq x \in X, f \in S_{X^{*}}$ is said to be a supporting functional at $x$ if $f(x)=\|x\|$. Let $J(x)=\left\{f \in S_{X^{*}}: f(x)=\right.$ $\|x\|\}, 0 \neq x \in X$, denote the collection of all supporting functionals at $x$. Note that for each $0 \neq x \in X$, the Hahn-Banach theorem ensures the existence of at least one supporting functional at $x$.

An element $x \in S_{X}$ is said to be a smooth point if $J(x)=\{f\}$ for some $f \in S_{X^{*}}$. Let $\operatorname{sm} S_{X}$ denote the collection of all smooth points of $S_{X}$. In particular if sm $S_{X}=S_{X}$, then $X$ is said to be a smooth space. Let $X$ be a Banach space with a norm \|\| . For every $\tau>0$, the modulus of smoothness is defined by

$$
\rho(\tau)=\sup \left\{\frac{\|x+\tau y\|+\|x-\tau y\|-2}{2}: x, y \in S_{X}\right\} .
$$

$(X,\|\|)$ is said to be a uniformly smooth space if $\lim _{\tau \rightarrow 0} \frac{\rho(\tau)}{\tau}=0$.

Let $X$ be a Banach space with a norm \|\| . For every $\varepsilon \in(0,2]$, the modulus of convexity is defined by

$$
\delta(\varepsilon)=\inf \left\{1-\frac{\|x+y\|}{2}: x, y \in B_{X},\|x-y\| \geq \varepsilon\right\} .
$$

$(X,\|\|)$ is said to be uniformly convex if $\delta(\varepsilon)>0$ for all $\varepsilon \in(0,2]$.

It is well known that a Banach space $(X,\|\|)$ is uniformly smooth if and only if its dual space $\left(X^{*},\|\|^{*}\right)$ is uniformly convex (see [10] for more details).

For $x, y \in X$, we say that $x$ is Birkhoff-James orthogonal to $y[2,7]$, written as $x \perp_{B} y$, if $\|x+\lambda y\| \geq\|x\|$ for all $\lambda \in \mathbb{R}$. In [7, Theorem 2.1], James proved that if $0 \neq x \in X, y \in X$, then $x \perp_{B} y$ if and only if there exists $f \in J(x)$ such that $f(y)=0$. We will use the notations $x^{\perp}=\left\{y \in X: x \perp_{B} y\right\}$ and ${ }^{\perp} x=\left\{y \in X: y \perp_{B} x\right\}$. Sain [12] characterized the Birkhoff-James orthogonality of linear operators between finite-dimensional Banach spaces by introducing the notions of the positive part of $x$, denoted by $x^{+}$, and the negative part of $x$, denoted by $x^{-}$, for an element $x \in X$. For any element 
$y \in X$, we say that $y \in x^{+}\left(y \in x^{-}\right)$if $\|x+\lambda y\| \geq\|x\|$ for all $\lambda \geq 0(\lambda \leq 0)$. It is easy to see that $x^{\perp}=x^{+} \cap x^{-}$.

Dragomir [6] defined an approximate Birkhoff-James orthogonality as follows. Let $\varepsilon \in[0,1)$ and let $x, y \in X$; then $x$ is said to be approximately Birkhoff-James orthogonal to $y$ if $\|x+\lambda y\| \geq(1-\varepsilon)\|x\|$ for all $\lambda \in \mathbb{R}$. Later on, Chmieliński [3] slightly modified the definition given by Dragomir as follows. Let $\varepsilon \in[0,1)$ and let $x, y \in X$. Then $x$ is said to be approximately BirkhoffJames orthogonal to $y$, written as $x \perp_{D}^{\varepsilon} y$, if and only if $\|x+\lambda y\| \geq \sqrt{1-\varepsilon^{2}}\|x\|$ for all $\lambda \in \mathbb{R}$. Due to this modification, in case of a Hilbert space, the present notion of the approximate orthogonality coincides exactly with the usual notion of the $\varepsilon$-orthogonality: $|\langle x, y\rangle| \leq \varepsilon\|x\|\|y\|$. In [9, Lemma 3.2], Mal et al. proved that

$$
x \perp_{D}^{\varepsilon} y \quad \Leftrightarrow \quad \exists f \in S_{X^{*}}:|f(x)| \geq \sqrt{1-\varepsilon^{2}}\|x\| \quad \text { and } \quad f(y)=0 .
$$

Chmieliński [3] defined a variation of approximate Birkhoff-James orthogonality. Given $x, y \in X$ and $\varepsilon \in[0,1), x$ is said to be approximately orthogonal to $y$, written as $x \perp_{B}^{\varepsilon} y$, if $\|x+\lambda y\|^{2} \geq\|x\|^{2}-2 \varepsilon\|x\|\|\lambda y\|$ for all $\lambda \in \mathbb{R}$. Later, in [4, Theorems 2.2 and 2.3], Chmieliński et al. gave two characterizations of this approximate orthogonality:

$$
\begin{gathered}
x \perp_{B}^{\varepsilon} y \quad \Leftrightarrow \quad \exists z \in \operatorname{span}\{x, y\}: x \perp_{B} z, \quad \text { and } \quad\|z-y\| \leq \varepsilon\|y\| ; \\
x \perp_{B}^{\varepsilon} y \quad \Leftrightarrow \quad \exists f \in J(x):|f(y)| \leq \varepsilon\|y\| .
\end{gathered}
$$

Given $x, y \in X$ and $\varepsilon \in[0,1)$, we will write $x \perp_{D}^{\varepsilon^{*}} y\left(x \perp_{B}^{\varepsilon^{*}} y\right)$ if $x \perp_{D}^{\varepsilon} y$ $\left(x \perp_{B}^{\varepsilon} y\right)$ but $x \not \perp_{D}^{\varepsilon_{1}} y\left(x \not \perp_{B}^{\varepsilon_{1}} y\right)$ for any $0 \leq \varepsilon_{1}<\varepsilon$.

In general, the orthogonality relation between two elements $x, y \in X$ need not be symmetric. In other words, for any two elements $x, y \in X, x \perp_{B} y$ does not necessarily imply $y \perp_{B} x$. James [8] proved that if $\operatorname{dim} X \geq 3$ and the Birkhoff--James orthogonality is symmetric, then the norm is induced by an inner product. For more details on the recent study of these notions of approximate Birkhoff-James orthogonality see $[14,15]$.

In [5], Chmieliński and Wójcik defined the following notion of approximate symmetry of the Birkhoff-James orthogonality in a normed linear space.

Definition 1.1 Let $X$ be a normed linear space. Then the Birkhoff-James orthogonality is approximately symmetric if there exists $\varepsilon \in[0,1)$ such that whenever $x, y \in X$ and $x \perp_{B} y$, it follows that $y \perp_{B}^{\varepsilon} x$.

The above definition is global in the sense that $\varepsilon$ is independent of $x$ and $y$.

In this paper we will work with both of the above mentioned notions of approximate Birkhoff-James orthogonality. To avoid any confusion we will call the above notion of approximate symmetry an approximate symmetry of the Birkhoff-James orthogonality in the sense of Chmielinski or shortly: $C$-approximate symmetry of the Birkhoff-James orthogonality. 
In [5], the authors gave an example of a Banach space where the BirkhoffJames orthogonality is not C-approximately symmetric. In the present article we will study this example in more detail. The following definition allows us to study local versions of the C-approximate symmetry of the Birkhoff--James orthogonality.

Definition 1.2 Let $X$ be a normed linear space and let $x \in X$. We say that $x$ is $C$-approximately left-symmetric ( $C$-approximately right-symmetric) if there exists $\varepsilon_{x} \in[0,1)$ such that whenever $y \in X$ and $x \perp_{B} y\left(y \perp_{B} x\right)$, it follows that $y \perp_{B}^{\varepsilon_{x}} x\left(x \perp_{B}^{\varepsilon_{x}} y\right)$.

For $\mathcal{A} \subseteq X$ we say that the Birkhoff-James orthogonality is $\mathrm{C}$-approximately symmetric on $\mathcal{A}$ if there exists $\varepsilon \in[0,1)$ such that whenever $x, y \in \mathcal{A}$ and $x \perp_{B} y$, it follows that $y \perp_{B}^{\varepsilon} x$.

Let $\mathcal{A} \subseteq X$ and let $x \in S_{X}$. We say that $x$ is C-approximately leftsymmetric (C-approximately right-symmetric) on $\mathcal{A}$ if there exists $\varepsilon_{x} \in[0,1)$ such that whenever $y \in \mathcal{A}$ and $x \perp_{B} y\left(y \perp_{B} x\right)$, it follows that $y \perp_{B}^{\varepsilon_{x}} x$ $\left(x \perp_{B}^{\varepsilon_{x}} y\right)$.

Now, with respect to the Dragomir's definition, we define the following analogous versions of approximate symmetry considered in Definitions 1.1 and 1.2 .

Definition 1.3 Let $X$ be a normed linear space. We say that the BirkhoffJames orthogonality is approximately symmetric in the sense of Dragomir, shortly: the Birkhoff-James orthogonality is D-approximately symmetric, if there exists $\varepsilon \in[0,1)$ such that whenever $x, y \in X$ and $x \perp_{B} y$, it follows that $y \perp_{D}^{\varepsilon} x$. For $x \in X$, we define $x$ to be $\mathrm{D}$-approximately left-symmetric (Dapproximately right-symmetric), if there exists $\varepsilon_{x} \in[0,1)$ such that whenever $y \in X$ and $x \perp_{B} y\left(y \perp_{B} x\right)$, it follows that $y \perp_{D}^{\varepsilon_{x}} x\left(x \perp_{D}^{\varepsilon_{x}} y\right)$.

Observe that we can restrict ourselves to norm-one elements by virtue of the homogeneity of all the notions of orthogonality and approximate orthogonality introduced here.

To study the C-approximate left-symmetry and the C-approximate rightsymmetry of elements of a normed linear space $X$, we define the following property. We say that the local property (P) holds for $x \in S_{X}$ if

$$
x^{\perp} \cap \mathscr{A}(x)=\emptyset,
$$

where $\mathscr{A}(x)$ is the collection of all those elements $y \in S_{X}$ for which given any $f \in J(y)$, either $f$ or $-f$ is in $J(x)$.

We say that the property $(\mathrm{P})$ holds for a normed linear space $X$ if the local property $(\mathrm{P})$ holds for each $x \in S_{X}$, that is,

$$
\text { for all } x \in S_{X} \text { : the local property (P) holds. }
$$

If $\mathcal{A} \subseteq S_{X}$ and $x \in S_{X}$, then we say that the local property (P) holds for $x$ on $\mathcal{A}$ if $x^{\perp} \cap \mathscr{A}(x) \cap \mathcal{A}=\emptyset$. 
It follows trivially that the local property $(\mathrm{P})$ holds for each $x \in \operatorname{sm} S_{X}$. We will prove that the local property $(\mathrm{P})$ for an $x \in S_{X}$ is equivalent to the C-approximate left-symmetry of $x$ in the local sense, that is, the local property (P) holds for $x \in S_{X}$ if and only if for $y \in x^{\perp} \cap S_{X}$ there exists $\varepsilon_{x, y} \in[0,1)$ such that $y \perp_{B}^{\varepsilon_{x, y}} x$.

To study polyhedral Banach spaces, we recall the following definitions from [13] which are relevant to our work:

Definition 1.4 Let $X$ be an $n$-dimensional Banach space. A polyhedron $P$ is a non-empty compact subset of $X$ which is an intersection of finitely many closed half-spaces of $X$, that means $P=\cap_{i=1}^{r} M_{i}$, where $M_{i}$ are closed halfspaces in $X$ and $r \in \mathbb{N}$. The dimension of the polyhedron $P$ is defined to be the dimension of the subspace generated by the differences $x-y$ of vectors $x, y \in P$.

An $n$-dimensional Banach space $X$ is said to be a polyhedral Banach space if $B_{X}$ contains only finitely many extreme points, or, equivalently, if $S_{X}$ is a polyhedron.

Definition 1.5 Let $X$ be an $n$-dimensional Banach space. A polyhedron $Q \subseteq X$ is said to be a face of the polyhedron $P \subseteq X$ if either $Q=P$ or if we can write $Q=P \cap \delta M$, where $M$ is a closed half-space in $X$ containing $P$ and $\delta M$ denotes the boundary of $M$. If the dimension of $Q$ is $i$, then $Q$ is called an $i$-face of $P$. $(n-1)$-faces are called facets of $P$ and 1 -faces of $P$ are called edges of $P$.

Definition 1.6 Let $X$ be a finite-dimensional polyhedral Banach space and let $F$ be a facet of the unit ball $B_{X}$. A functional $f \in S_{X^{*}}$ is said to be a supporting functional corresponding to the facet $F$ of the unit ball $B_{X}$ if the following two conditions are satisfied:

(a) $f$ attains its norm at some point $v$ of $F$.

(b) $F=(v+k e r f) \cap S_{X}$.

It is easy to see that there is a unique hyperspace $H$ such that an affine hyperplane parallel to $H$ contains the facet $F$ of the unit ball $B_{X}$. Moreover, there exists a unique norm-one functional $f$, such that $f$ attains its norm on $F$ and ker $f=H$. In particular, $f$ is a supporting functional to $B_{X}$ at every point of $F$.

Two elements $x, y \in \operatorname{Ext} B_{X}$ of an $n$-dimensional polyhedral Banach space $X$ are said to be adjacent if $\|t x+(1-t) y\|=1$ for all $t \in[0,1]$.

Given normed linear spaces $X, Y$, by $\mathcal{B}(X, Y)(\mathcal{K}(X, Y))$ we denote the space of all bounded (compact) linear operators from $X$ to $Y$. A bounded linear operator $T \in \mathcal{B}(X, Y)$ is said to attain its norm at $x \in S_{X}$ if $\|T x\|=\|T\|$. Let $M_{T}=\left\{x \in S_{X}:\|T x\|=\|T\|\right\}$ be the collection of all norm attaining elements of $T$. If $X$ is a reflexive Banach space and $T \in \mathcal{K}(X, Y)$, then $M_{T} \neq \emptyset$ (see [1] for details). 
The article is organized as follows. In Sect. 2, we study the D-approximate symmetry of the Birkhoff--James orthogonality. For this notion the results we are able to obtain are of the highest level of generality. In particular, we prove that in all finite-dimensional Banach spaces, the Birkhoff-James orthogonality is always D-approximately symmetric.

In Sect. 3, we study the C-approximate symmetry of the Birkhoff-James orthogonality. We prove that in finite-dimensional polyhedral Banach spaces, the C-approximate symmetry of the Birkhoff-James orthogonality is equivalent to the local property $(\mathrm{P})$ of all elements of Ext $B_{X}$. Apparently, the results in this section are less general than in Sect. 2 . It is caused by the fact that the notion of the C-approximate symmetry essentially differs from the D-approximate one and not all properties remain true. Thus we need to use more subtle methods which usually involve additional assumptions.

In Sect. 4, we study the C-approximate symmetry of the Birkhoff-James orthogonality for two-dimensional Banach spaces. Even in this case, establishing a satisfactory characterization of the C-approximate symmetry is challenging. To this aim, we introduce a new property, namely property (P1). We show that for any finite-dimensional polyhedral Banach space with property (P1), local property $(\mathrm{P})$ also holds for each element. We also show that the converse is true for any two-dimensional polyhedral Banach spaces but in general it need not be true. We show that in a two-dimensional regular polyhedral Banach space with $2 n$ vertices, where $n \geq 3$, the Birkhoff-James orthogonality is C-approximately symmetric. We provide an example to show that the regularity condition in this case cannot be dropped.

\section{D-approximate Symmetry of the Birkhoff-James Orthogonality}

In [5], Chmieliński and Wójcik proved that in uniformly convex Banach spaces and finite-dimensional smooth Banach spaces, the Birkhoff-James orthogonality is C-approximately symmetric. Our main aim in this section is to prove that for any finite-dimensional Banach space, the Birkhoff-James orthogonality is D-approximately symmetric. To achieve this aim, we first prove the following results.

Theorem 2.1 Let $X$ be a normed linear space and let $x, y \in S_{X}$ with $x \perp_{D}^{\varepsilon} y$ for some $\varepsilon \in[0,1)$. Then there exist $\varepsilon_{1}, \varepsilon_{2}>0, \varepsilon_{3} \in(0,1)$ such that $z \perp_{D}^{\varepsilon_{3}} w$ for all $z \in B\left(x, \varepsilon_{1}\right) \cap S_{X}, w \in B\left(y, \varepsilon_{2}\right) \cap S_{X}$.

Proof. Let $\varepsilon_{1}>0$ be such that $\sqrt{1-\varepsilon^{2}}-\varepsilon_{1}>0$. If $z \in B\left(x, \varepsilon_{1}\right) \cap S_{X}$, then

$$
\|z+\lambda y\|=\|z-x+x+\lambda y\| \geq\|x+\lambda y\|-\|x-z\| \geq \sqrt{1-\varepsilon^{2}}-\varepsilon_{1} .
$$

Thus for all $z \in B\left(x, \varepsilon_{1}\right) \cap S_{X}$, we have, $z \perp_{D}^{\delta} y$ where $\delta$ is such that $\sqrt{1-\varepsilon^{2}}-\varepsilon_{1}=\sqrt{1-\delta^{2}}$. 
If $|\lambda| \geq 2$, then for any $z_{1}, z_{2} \in S_{X}$, we have, $\left\|z_{1}+\lambda z_{2}\right\| \geq|\lambda|-1 \geq 1 \geq$ $1-\beta$ for all $\beta \in[0,1)$.

Choose $\varepsilon_{2}>0$ such that $\sqrt{1-\delta^{2}}-2 \varepsilon_{2}>0$. Now, if $\lambda \in \mathbb{R}$ with $|\lambda|<2$, then for any $z \in B\left(x, \varepsilon_{1}\right) \cap S_{X}$ and $w \in B\left(y, \varepsilon_{2}\right) \cap S_{X}$, we have,

$$
\|z+\lambda w\|=\|z+\lambda y+\lambda w-\lambda y\| \geq\|z+\lambda y\|-|\lambda|\|y-w\|>\sqrt{1-\delta^{2}}-2 \varepsilon_{2} .
$$

Thus for all $z \in B\left(x, \varepsilon_{1}\right) \cap S_{X}$ and $w \in B\left(y, \varepsilon_{2}\right) \cap S_{X}$, we have, $z \perp_{D}^{\varepsilon_{3}} w$ where $\varepsilon_{3}$ is such that $\sqrt{1-\delta^{2}}-2 \varepsilon_{2}=\sqrt{1-\varepsilon_{3}^{2}}$.

Our next result shows that given any two linearly independent elements $x, y \in S_{X}$ of a normed linear space $X$, we can always find an $\varepsilon \in[0,1)$ (depending on $x$ and $y$ ) such that $x \perp_{D}^{\varepsilon} y$.

Proposition 2.2 Let $X$ be a normed linear space and let $x, y \in S_{X}$ with $x \neq \pm y$. Then there exists $\varepsilon_{x, y} \in[0,1)$ such that $x \perp_{D}^{\varepsilon_{x, y}} y$.

Proof. Since $x, y \in S_{X}$ and $x \neq \pm y$, it follows that $x, y$ are linearly independent. Let $X_{0}=\operatorname{span}\{x, y\}$ and let $\left\{x^{*}, y^{*}\right\} \subseteq X_{0}^{*}$ be such that $\left\{x, y ; x^{*}, y^{*}\right\}$ is a biorthogonal system in $X_{0}$, where $x^{*}(x)=y^{*}(y)=1, x^{*}(y)=y^{*}(x)=0$. Now, if we take $f=\frac{x^{*}}{\left\|x^{*}\right\|}$, then $f \in S_{X_{0}^{*}}, f(x)=\frac{1}{\left\|x^{*}\right\|}$ and $f(y)=0$. Let $\hat{f}$ be a Hahn-Banach extension of $f$ to $X^{*}$. Then $\hat{f} \in S_{X^{*}}, \hat{f}(x)=\frac{1}{\left\|x^{*}\right\|}$ and $\hat{f}(y)=0$. If $\frac{1}{\left\|x^{*}\right\|}>1$, then for all $\varepsilon \in[0,1)$, we have, $\hat{f}(x) \geq \sqrt{1-\varepsilon^{2}}$. If $\frac{1}{\left\|x^{*}\right\|} \leq 1$, then we can find $\varepsilon \in[0,1)$ such that $\hat{f}(x) \geq \sqrt{1-\varepsilon^{2}}$. Thus (1.1) implies that for given $x, y \in S_{X}$, with $x \neq \pm y$, there exists $\varepsilon_{x, y} \in[0,1)$ such that $x \perp_{D}^{\varepsilon_{x, y}} y$.

Theorem 2.3 Let $X$ be a finite-dimensional Banach space. Then the BirkhoffJames orthogonality is D-approximately symmetric in $X$.

Proof. Let $x \in S_{X}$ and let $y \in x^{\perp} \cap S_{X}$. Then by Proposition 2.2, there exists $\varepsilon_{x, y} \in[0,1)$ such that $y \perp_{D}^{\varepsilon_{x, y}} x$. Let $\varepsilon_{x, y}^{*}$ be the infimum of all such $\varepsilon_{x, y}$. We claim that $\varepsilon:=\sup _{x \in S_{X}} \sup _{y \in x^{\perp} \cap S_{X}} \varepsilon_{x, y}^{*}<1$. If $\varepsilon=1$, then we can choose $\left\{x_{n}\right\}$, $\left\{y_{n}\right\} \subseteq S_{X}, \varepsilon_{n} \nearrow 1$ such that $x_{n} \perp_{B} y_{n}$ and $y_{n} \perp_{D}^{\varepsilon_{n}^{*}} x_{n}$. Since $S_{X}$ is compact, there exist convergent sub-sequences of $\left\{x_{n}\right\},\left\{y_{n}\right\}$ which we again denote by $\left\{x_{n}\right\}$ and $\left\{y_{n}\right\}$, respectively. Let $x_{0}, y_{0} \in S_{X}$ be such that $x_{n} \longrightarrow x_{0}$ and $y_{n} \longrightarrow y_{0}$. Then by continuity of the norm it follows that $y_{0} \in x_{0}^{\perp} \cap S_{X}$. Now from Proposition 2.2, it follows that $y_{0} \perp_{D}^{\varepsilon_{0}} x_{0}$ for some $\varepsilon_{0} \in[0,1)$. Using Theorem 2.1, we can find $\varepsilon_{1}, \varepsilon_{2}>0$ and $\varepsilon_{3} \in(0,1)$ such that $w \perp_{D}^{\varepsilon_{3}} z$ for all $z \in B\left(x_{0}, \varepsilon_{1}\right) \cap S_{X}$ and $w \in B\left(y_{0}, \varepsilon_{2}\right) \cap S_{X}$. Thus we can find $m \in \mathbb{N}$ such that $y_{n} \perp_{D}^{\varepsilon_{3}} x_{n}$ for all $n \geq m$. This leads to a contradiction as $y_{n} \perp_{D}^{\varepsilon_{n}^{*}} x_{n}$ for $\varepsilon_{n} \nearrow 1$. Thus $\varepsilon<1$ and the Birkhoff-James orthogonality is D-approximately symmetric in $X$. 
Remark 2.4 It follows from the above theorem that each element of a finitedimensional Banach space is both D-approximately left-symmetric and Dapproximately right-symmetric.

We will use the following result from [11] in the proof of the next result.

Theorem 2.5 [11, Theorem 2.1] Let $X$ be a reflexive Banach space and let $Y$ be a normed linear space. Let $T, A \in \mathcal{K}(X, Y)$ with $\|T\|=\|A\|=1$. Then $T \perp_{D}^{\varepsilon} A$ for $\varepsilon \in[0,1)$ if and only if either $(a)$ or $(b)$ holds.

(a) There exists $x \in M_{T}$ such that $A x \in(T x)^{+}$and for each $\lambda \in(-1-$ $\left.\sqrt{1-\varepsilon^{2}},-1+\sqrt{1-\varepsilon^{2}}\right)$, there exists $x_{\lambda} \in S_{X}$ such that $\left\|T x_{\lambda}+\lambda A x_{\lambda}\right\| \geq$ $\sqrt{1-\varepsilon^{2}}$.

(b) There exists $y \in M_{T}$ such that $A y \in(T y)^{-}$and for each $\lambda \in(1-$ $\left.\sqrt{1-\varepsilon^{2}}, 1+\sqrt{1-\varepsilon^{2}}\right)$, there exists $y_{\lambda} \in S_{X}$ such that $\left\|T y_{\lambda}+\lambda A y_{\lambda}\right\| \geq$ $\sqrt{1-\varepsilon^{2}}$.

Let $X$ be a reflexive Banach space and let $Y$ be a normed linear space. Let $T, A \in S_{\mathcal{K}(X, Y)}$ be such that $T \perp_{B} A$. Then by Proposition 2.2, there exists $\varepsilon \in[0,1)$ such that $A \perp_{D}^{\varepsilon} T$. We now estimate the infimum of such $\varepsilon$ 's.

Theorem 2.6 Let $X$ be a reflexive Banach space and $Y$ a normed linear space. Suppose that $T, A \in \mathcal{K}(X, Y)$ with $\|T\|=\|A\|=1$ and that the set $\mathcal{A}=\{x \in$ $S_{X}: T x \neq \lambda A x$ for all $\left.\lambda \in \mathbb{R}\right\}$ is nonempty. If $A \perp_{B} T$, then $T \perp_{D}^{\varepsilon} A$, where $\sqrt{1-\varepsilon^{2}}=\sup _{x \in \mathcal{A}} \inf _{\lambda \in \mathbb{R}}\|T x+\lambda A x\|$.

Proof. Let $x_{0} \in \mathcal{A}$. Then $T x_{0} \neq 0$ and by continuity of the function $f(\lambda)=$ $\left\|T x_{0}+\lambda A x_{0}\right\|, \lambda \in \mathbb{R}$ and the fact that $f(\lambda) \longrightarrow \infty$ as $\lambda \longrightarrow \pm \infty$, it follows that $\inf _{\lambda}\left\|T x_{0}+\lambda A x_{0}\right\|>0$. Also, $\inf _{\lambda}\left\|T x_{0}+\lambda A x_{0}\right\| \leq\left\|T x_{0}\right\| \leq 1$. Let $\varepsilon_{x_{0}} \in$ $[0,1)$ be such that $\inf _{\lambda}\left\|T x_{0}+\lambda A x_{0}\right\|=\sqrt{1-\varepsilon_{x_{0}}^{2}}$. If $x \in X$, then it follows from [12, Proposition 2.1] that either $A x \in(T x)^{+}$or $A x \in(T x)^{-}$. Since $X$ is a reflexive Banach space and $T \in \mathcal{K}(X, Y)$, it follows that $M_{T} \neq \emptyset$. Now, by using Theorem 2.5, we get $T \perp_{D}^{\varepsilon_{x_{0}}} A$. If we fix $\alpha=\sup _{x \in \mathcal{A}} \inf _{\lambda \in \mathbb{R}}\|T x+\lambda A x\|$, then clearly $\alpha \in(0,1]$. Let $\varepsilon \in[0,1)$ be such that $\alpha=\sqrt{1-\varepsilon^{2}}$. Then $T \perp_{D}^{\varepsilon} A$ and this completes the proof.

Remark 2.7 The proof of the above theorem suggests that if $x_{0} \in \mathcal{A}$ and $\inf _{\lambda}\left\|T x_{0}+\lambda A x_{0}\right\|=\sqrt{1-\varepsilon_{x_{0}}^{2}}$, then $T \perp_{D}^{\varepsilon_{x_{0}}} A$. Thus $\sqrt{1-\varepsilon^{2}}=\sup _{x \in \mathcal{A}} \inf _{\lambda \in \mathbb{R}}$ $\|T x+\lambda A x\|$ provides the best possible estimate for $\varepsilon \in[0,1)$ such that $T \perp_{D}^{\varepsilon} A$.

As an application of the above theorem, for finite-dimensional spaces $X$, $Y$ and operators $T, A \in S_{\mathcal{B}(X, Y)}$ with $A \perp_{B} T$, we now obtain an estimate of $\varepsilon$ such that $T \perp_{D}^{\varepsilon} A$.

Theorem 2.8 Let $X, Y$ be finite-dimensional Banach spaces. Let $T, A \in \mathcal{B}(X, Y)$ with $\|T\|=\|A\|=1$ and let $\mathcal{A}=\left\{x \in S_{X}: T x \neq \lambda A x\right.$ for all $\left.\lambda \in \mathbb{R}\right\}$. If $A \perp_{B} T$, then $T \perp_{D}^{\varepsilon} A$, where $\sqrt{1-\varepsilon^{2}}=\sup _{x \in \mathcal{A}} \inf _{\lambda \in \mathbb{R}}\|T x+\lambda A x\|$. 
Proof. In order to apply Theorem 2.6, we need to show that $\mathcal{A} \neq \emptyset$. Suppose on the contrary that $\mathcal{A}=\emptyset$. Then for each $x \in S_{X}$, there exists $\lambda_{x} \in \mathbb{R}$ such that $T x=\lambda_{x} A x$. Clearly, $A \perp_{B} T$ implies that there does not exist $\lambda \in \mathbb{R}$ such that $T x=\lambda A x$ for all $x \in X$. We now consider the following two cases.

Let rank $A \geq 2$ and let $\left\{A x_{1}, \ldots, A x_{k}\right\}$ be a basis for range $A$, where $x_{1}, \ldots, x_{k} \in S_{X}, 2 \leq k \leq n$, where $\operatorname{dim} X=n$. Let $\left\{x_{1}, \ldots, x_{k}, x_{k+1}, \ldots, x_{n}\right\}$ be a basis for $X$, where $\left\{x_{k+1}, \ldots, x_{n}\right\} \subseteq S_{X}$ is a basis for ker $A$.

In this case there are following two possibilities:

(i) there exist $1 \leq i, j \leq k$ such that $T x_{i}=\lambda_{x_{i}} A x_{i}$ and $T x_{j}=\lambda_{x_{j}} A x_{j}$ for $\lambda_{x_{i}} \neq \lambda_{x_{j}}$,

(ii) there exists a $\lambda \in \mathbb{R}$ such that $T x_{i}=\lambda A x_{i}$ for each $1 \leq i \leq k$.

First consider the case $(i)$. In this case

$$
T\left(\frac{x_{i}+x_{j}}{\left\|x_{i}+x_{j}\right\|}\right)=\frac{\lambda_{x_{i}} A x_{i}+\lambda_{x_{j}} A x_{j}}{\left\|x_{i}+x_{j}\right\|} .
$$

Using the assumption $\mathcal{A}=\emptyset$, let $\lambda \in \mathbb{R}$ be such that

$$
T\left(\frac{x_{i}+x_{j}}{\left\|x_{i}+x_{j}\right\|}\right)=\lambda A\left(\frac{x_{i}+x_{j}}{\left\|x_{i}+x_{j}\right\|}\right) .
$$

Thus $\left(\lambda_{x_{i}}-\lambda\right) A x_{i}+\left(\lambda_{x_{j}}-\lambda\right) A x_{j}=0$ and this proves that $\lambda=\lambda_{x_{i}}=\lambda_{x_{j}}$. This leads to a contradiction as $\lambda_{x_{i}} \neq \lambda_{x_{j}}$.

Now, we will consider the case $(i i)$ as above. In this case $x_{i_{0}} \notin \operatorname{ker} T$ for at least one $i_{0}, k+1 \leq i_{0} \leq n$, otherwise $T=\lambda A$. Clearly $T x_{i_{0}} \neq \lambda A x_{i_{0}}$ for all $\lambda \in \mathbb{R}$. This contradicts that $\mathcal{A}=\emptyset$. Thus if rank $A \geq 2$, then $\mathcal{A} \neq \emptyset$.

Now, consider the case when $\operatorname{rank} A=1$. Let range $A=\operatorname{span}\left\{A x_{1}\right\}$ where $x_{1} \in S_{X}$ and $\left\{x_{2}, \ldots, x_{n}\right\} \subseteq S_{X}$ be a basis for ker $A$. By the assumption $\mathcal{A}=\emptyset$ and thus $T x_{1}=\lambda_{x_{1}} A x_{1}$ for some $\lambda_{x_{1}} \in \mathbb{R}$. Clearly, $A \perp_{B} T$ implies $x_{i_{0}} \notin \operatorname{ker} T$ for at least one $i_{0}, 2 \leq i_{0} \leq n$. This implies $T x_{i_{0}} \neq \lambda A x_{i_{0}}$ for all $\lambda \in \mathbb{R}$. This contradicts that $\mathcal{A}=\emptyset$ and thus in this case also $\mathcal{A} \neq \emptyset$. Now, the result follows from Theorem 2.6.

The above theorem can be extended to compact operators on a reflexive Banach space, under the additional assumption of injectivity of $A$ or $T$.

Theorem 2.9 Let $X$ be a reflexive Banach space and $Y$ any normed linear space. Assume that $T, A \in \mathcal{K}(X, Y)$ with $\|T\|=\|A\|=1$ and either $A$ or $T$ is one to one operator. Define $\mathcal{A}=\left\{x \in S_{X}: T x \neq \lambda A x\right.$ for all $\left.\lambda \in \mathbb{R}\right\}$. If $A \perp_{B} T$, then $T \perp_{D}^{\varepsilon} A$, where $\sqrt{1-\varepsilon^{2}}=\sup _{x \in \mathcal{A}} \inf _{\lambda \in \mathbb{R}}\|T x+\lambda A x\|$.

Proof. To prove the result we need to show that $\mathcal{A} \neq \emptyset$. Suppose on the contrary that $\mathcal{A}=\emptyset$. Then for each $x \in S_{X}$, there exists $\lambda_{x} \in \mathbb{R}$ such that $T x=\lambda_{x} A x$. Clearly $A \perp_{B} T$ implies that there exist $x, y \in S_{X}$ such that

$$
T x=\lambda_{x} A x \quad \text { and } \quad T y=\lambda_{y} A y
$$

for $\lambda_{x} \neq \lambda_{y}$. This implies $x$ and $y$ are linearly independent in $X$. 
Let $\lambda \in \mathbb{R}$ be such that

$$
T\left(\frac{x+y}{\|x+y\|}\right)=\lambda A\left(\frac{x+y}{\|x+y\|}\right) .
$$

Also, we have,

$$
T\left(\frac{x+y}{\|x+y\|}\right)=\frac{\lambda_{x} A x+\lambda_{y} A y}{\|x+y\|} .
$$

Let us first assume that $A$ is one to one operator. Now, using (2.2), (2.3) we have $A\left(\left(\lambda_{x}-\lambda\right) x+\left(\lambda_{y}-\lambda\right) y\right)=0$ and using the assumption that $A$ is one to one we get $\left(\lambda_{x}-\lambda\right) x+\left(\lambda_{y}-\lambda\right) y=0$. It follows from the linear independence of $x, y$ that $\lambda_{x}=\lambda=\lambda_{y}$. But this leads to a contradiction as $\lambda_{x} \neq \lambda_{y}$. This implies that $\mathcal{A} \neq \emptyset$. Thus in this case the result follows from Theorem 2.6.

Now, we assume that $T$ is one to one operator. It follows from this assumption on $T$ that in (2.1) $\lambda_{x}, \lambda_{y} \neq 0$ and also in (2.2), we have $\lambda \neq 0$. After rewriting (2.2) and using (2.1), we get,

$$
\begin{aligned}
\frac{1}{\lambda} T\left(\frac{x+y}{\|x+y\|}\right) & =A\left(\frac{x+y}{\|x+y\|}\right), \\
\frac{\frac{1}{\lambda_{x}} T x+\frac{1}{\lambda_{y}} T y}{\|x+y\|} & =A\left(\frac{x+y}{\|x+y\|}\right) .
\end{aligned}
$$

Thus $T\left(\left(\frac{1}{\lambda_{x}}-\frac{1}{\lambda}\right) x+\left(\frac{1}{\lambda_{y}}-\frac{1}{\lambda}\right) y\right)=0$ and using the assumption that $T$ is one to one we get $\left(\frac{1}{\lambda_{x}}-\frac{1}{\lambda}\right) x+\left(\frac{1}{\lambda_{y}}-\frac{1}{\lambda}\right) y=0$. Now, the result follows from the similar arguments as those used in the previous case.

\section{C-approximate Symmetry of the Birkhoff-James Orthogonality}

It was observed in [5], that in $\left(\mathbb{R}^{2},\|\|_{\infty}\right)$ the Birkhoff-James orthogonality is not C-approximately symmetric. In the following proposition we study the $\mathrm{C}$-approximate left-symmetry and the $\mathrm{C}$-approximate right-symmetry of elements of $\left(\mathbb{R}^{n},\|\|_{\infty}\right)$ in detail. In particular, the following result illustrates that in the local sense, the C-approximate left-symmetry is not equivalent to the C-approximate right-symmetry of the Birkhoff-James orthogonality. It is well known that the dual of $\left(\mathbb{R}^{n},\|\|_{\infty}\right)$ can be identified with $\left(\mathbb{R}^{n},\|\|_{1}\right)$, where the dual action is given by $f(x)=\sum_{i=1}^{n} f_{i} x_{i}$ for all $x=\left(x_{1}, \ldots, x_{n}\right) \in\left(\mathbb{R}^{n},\|\|_{\infty}\right)$ and $f=\left(f_{1}, \ldots, f_{n}\right) \in\left(\mathbb{R}^{n},\|\|_{1}\right)$. If $t \in \mathbb{R}$, then sgn $t$ denotes the sign function, that is, $\operatorname{sgn} t=\frac{t}{|t|}$ for $t \neq 0$ and $\operatorname{sgn} 0=0$.

Proposition 3.1 Let $X=\left(\mathbb{R}^{n},\|\|_{\infty}\right)$. Then

(i) any smooth point $x \in S_{X}$ is $C$-approximately left-symmetric but not $C$ approximately right-symmetric;

(ii) any extreme point $x$ of $S_{X}$ is C-approximately right-symmetric but not C-approximately left-symmetric. 
Proof. Observe that from the symmetry of $S_{X}$, it is sufficient to prove the result for any one of the extreme points and smooth points of $S_{X}$.

(i) Let $x=\left(1, x_{2}, \ldots, x_{n}\right) \in S_{X}$ be a smooth point. Then $\left|x_{i}\right|<1$ for all $2 \leq i \leq n$ and $J(x)=\{f\}$ where $f=(1,0, \ldots, 0) \in S_{X^{*}}$. Let $y=$ $\left(y_{1}, y_{2}, \ldots, y_{n}\right) \in S_{X}$ be such that $x \perp_{B} y$. Then by using (1.3), it follows that $y_{1}=0$. As $y \in S_{X}$, there exists $2 \leq i_{0} \leq n$ such that $\left|y_{i_{0}}\right|=1$. Let $g=\left(0,0, \ldots, 0, y_{i_{0}}, 0, \ldots, 0\right) \in S_{X^{*}}$, where $y_{i_{0}}$ is the $i_{0}$-th co-ordinate. Then $g \in J(y)$ and $|g(x)|=\left|x_{i_{0}}\right|<1$. Thus (1.3) implies that $y \perp_{B}^{\varepsilon_{0}} x$, where $\varepsilon_{0}=\left|x_{i_{0}}\right|$. Now, if we take $\varepsilon=\max _{2 \leq i \leq n}\left|x_{i}\right|$, then $\varepsilon \in[0,1)$ and $y \perp_{B}^{\varepsilon} x$ whenever $x \perp_{B} y$. Hence $x$ is C-approximately left-symmetric.

Now, we show that $x$ is not C-approximately right-symmetric. If $x_{i}=0$ for all $2 \leq i \leq n$, then $z \perp_{B} x$, where $z=(1,1, \ldots, 1)$. As $|f(z)|=1$, there does not exist any $\varepsilon \in[0,1)$ such that $x \perp_{B}^{\varepsilon} z$. Without loss of generality we now assume that $x_{2} \neq 0$. Let $w=\left(1,-\operatorname{sgn} x_{2}, x_{3}, \ldots, x_{n}\right) \in S_{X}$. Then for any $\lambda \geq 0$ we have $\|w+\lambda x\| \geq|1+\lambda| \geq 1$. Also, for any $\lambda<0$, we have $\|w+\lambda x\| \geq\left|-\operatorname{sgn} x_{2}+\lambda x_{2}\right|>1$.

This shows that $w \perp_{B} x$. As $|f(w)|=1$, there does not exist any $\varepsilon \in[0,1)$ such that $x \perp_{B}^{\varepsilon} w$. Thus, (1.3) implies that $x$ is not C-approximately rightsymmetric. Figure 1, given below, illustrates this situation for $n=2$.

(ii) Consider $x=(1,1, \ldots, 1) \in \operatorname{Ext} B_{X}$. It follows from the arguments of $(i)$ that $x$ is not C-approximately left-symmetric.

We now prove that $x$ is C-approximately right-symmetric. Consider $y=$ $\left(y_{1}, y_{2}, \ldots, y_{n}\right) \in S_{X}$ such that $y \perp_{B} x$. Since $y \in S_{X}$, there exists $1 \leq i \leq n$ such that $\left|y_{i}\right|=1$. Let $\left\{i_{1}, i_{2}, \ldots, i_{k}\right\} \subseteq\{1,2, \ldots, n\}$ be a maximal subset such that $\left|y_{i_{j}}\right|=1$ for $1 \leq j \leq k$. We now claim that $k>1$. Suppose on the contrary that $k=1$. Then $y \in \operatorname{sm} S_{X}, J(y)=\{f\}$, where $f=(0,0, \ldots, 0,1,0, \ldots, 0) \in$ $S_{X^{*}}$ and 1 is the $i_{1}$-th co-ordinate. But $f(x) \neq 0$ and this contradicts that $y \perp_{B} x$.

We now claim that there exist $1 \leq l \neq m \leq k$ such that $y_{i_{l}}=-y_{i_{m}}$. If $y_{i_{l}}=y_{i_{m}}$ for all $1 \leq l, m \leq k$, then for sufficiently small absolute value $\lambda$, it is easy to see that $\|y+\lambda x\|=\left|y_{i_{1}}+\lambda\right|$. This clearly contradicts that $y \perp_{B} x$ and hence there exist $1 \leq l \neq m \leq k$ such that $y_{i_{l}}=-y_{i_{m}}$. Now, if we take $g=\left(0,0, \ldots, 0, \frac{1}{2}, 0, \ldots, 0, \frac{1}{2}, 0, \ldots, 0\right) \in S_{X^{*}}$, where $\frac{1}{2}$ is at $i_{1}$-th and $i_{m}$-th coordinates. Then $g \in J(x)$ and $g(y)=0$. This shows that $x$ is right-symmetric and hence C-approximately right-symmetric. Figure 2, given below, illustrates this situation for $n=2$.

Remark 3.2 The proof of the above proposition suggests that in $X=\left(\mathbb{R}^{n},\|\|_{\infty}\right)$ the C-approximate right-symmetry (the C-approximate left-symmetry) of any $x \in \operatorname{sm} S_{X}\left(x \in \operatorname{Ext} B_{X}\right)$ fails because there exists $y \in{ }^{\perp} x \cap S_{X}\left(y \in x^{\perp} \cap S_{X}\right)$ such that either $f \in J(y)$ or $-f \in J(y)(f \in J(x)$ or $-f \in J(x))$ where $J(x)=\{f\}(f \in J(y))$. 


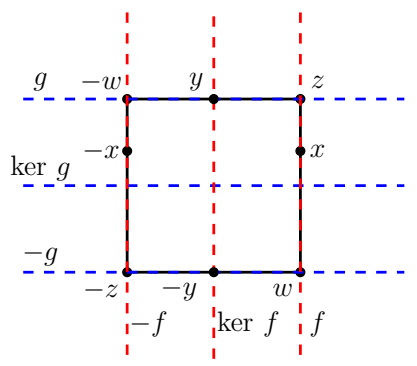

FiguRE 1. Approximate left-symmetry - not approximate right-symmetry.

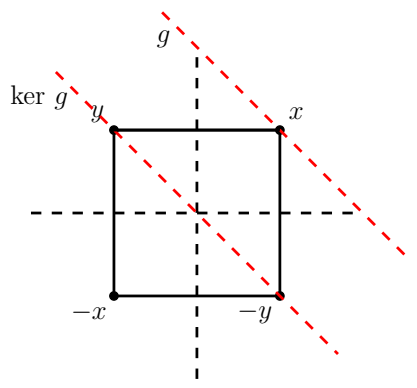

FiguRE 2. Approximate right-symmetry - not approximate left-symmetry.

The above remark is the main motivation behind considering the local property $(\mathrm{P})$ for $x \in S_{X}$ introduced in the first section. Recall that the local property $(\mathrm{P})$ holds for $x \in S_{X}$ if

$$
x^{\perp} \cap \mathscr{A}(x)=\emptyset,
$$

where $\mathscr{A}(x)$ is the collection of all those elements $y \in S_{X}$ for which given any $f \in J(y)$, either $f$ or $-f$ is in $J(x)$. Also, recall that the property (P) holds for a normed linear space $X$ if the local property $(\mathrm{P})$ holds for each $x \in S_{X}$.

We now show that in finite-dimensional Banach spaces, the local property (P) for all elements of Ext $B_{X}$ implies the property (P) globally for $X$.

Theorem 3.3 Let $X$ be a finite-dimensional Banach space and suppose that the local property $(\mathrm{P})$ holds for each $x \in \operatorname{Ext} B_{X}$. Then the local property $(\mathrm{P})$ holds for each $y \in S_{X}$.

Proof. It follows easily that in any normed linear space, the local property (P) holds for each smooth point. Thus, to prove the result we need to show that the local property $(\mathrm{P})$ holds for any $y \in S_{X} \backslash\left(\operatorname{sm} S_{X} \cup \operatorname{Ext} B_{X}\right)$. Let $y \in S_{X} \backslash\left(\operatorname{sm} S_{X} \cup \operatorname{Ext} B_{X}\right)$. Since $S_{X}$ is contained in the convex hull of 
Ext $B_{X}$, let $x_{1}, \ldots, x_{k} \in \operatorname{Ext} B_{X}, k \leq\left|\operatorname{Ext} B_{X}\right|$, be such that $y=\sum_{i=1}^{k} \alpha_{i} x_{i}$, $\alpha_{i}>0$ for all $1 \leq i \leq k$ and $\sum_{i=1}^{k} \alpha_{i}=1$.

Now, we claim that if $f \in J(y)$, then $f \in J\left(x_{i}\right)$ for all $1 \leq i \leq k$. Clearly, $\left|f\left(x_{i}\right)\right| \leq 1$ for all $1 \leq i \leq k$. Suppose on the contrary that $f\left(x_{j}\right)<1$ for some $1 \leq j \leq k$. Then

$$
1=f(y)=\sum_{i=1}^{k} \alpha_{i} f\left(x_{i}\right)<\sum_{i=1}^{k} \alpha_{i}=1 .
$$

This clearly leads to a contradiction and thus if $f \in J(y)$, then $f \in J\left(x_{i}\right)$ for all $1 \leq i \leq k$.

Let $z \in y^{\perp} \cap S_{X}$. Then there exists $g \in J(y)$ such that $g(z)=0$. But $g \in J\left(x_{i}\right)$ for all $1 \leq i \leq k$; this gives $x_{i} \perp_{B} z$ for all $1 \leq i \leq k$.

We now claim that there exists some $g_{0} \in J(z)$ such that $\left|g_{0}(y)\right|<1$. Suppose on the contrary that for any $g \in J(z)$ we have $|g(y)|=1$, that is, for any $g \in J(z)$ either $g$ or $-g$ is in $J(y)$. Thus for any $g \in J(z)$ either $g$ or $-g$ is in $J\left(x_{i}\right)$ for all $1 \leq i \leq k$. This clearly contradicts the local property $(\mathrm{P})$ of $x_{i}$, $1 \leq i \leq k$. Thus there exists some $g_{0} \in J(z)$ such that $\left|g_{0}(y)\right|<1$ and hence the local property $(\mathrm{P})$ of $y$ follows.

The next result shows that the local property $(\mathrm{P})$ of $x \in S_{X}$ is equivalent to the C-approximate left-symmetry of $x$ in the local sense.

Lemma 3.4 Let $X$ be a normed linear space. Then the local property $(\mathrm{P})$ holds for $x \in S_{X}$ if and only if for $y \in x^{\perp} \cap S_{X}$, there exists $\varepsilon_{x, y} \in[0,1)$ such that $y \perp_{B}^{\varepsilon_{x, y}} x$.

Proof. We first prove the necessary part of the lemma. Suppose on the contrary that there exists $y \in x^{\perp} \cap S_{X}$ such that $y \not \chi_{B}^{\varepsilon} x$ for any $\varepsilon \in[0,1)$. Clearly, if $f \in J(y)$, then $|f(x)| \leq 1$. Thus for all $f \in J(y)$ we have $|f(x)|=1$ and consequently either $f \in J(x)$ or $-f \in J(x)$. This contradicts that the local property $(\mathrm{P})$ holds for $x$ and thus the necessary part follows.

We now prove the sufficient part of the lemma. Let $y \in x^{\perp} \cap S_{X}$. It follows from the assumption that $y \perp_{B}^{\varepsilon_{x, y}} x$ and, equivalently, there exists $f \in J(y)$ such that $|f(x)| \leq \varepsilon_{x, y}<1$, hence $\pm f \notin J(x)$. Thus $y \notin \mathscr{A}(x)$ and the local property (P) of $x$ follows.

Observe that in the proof of Theorem 2.1, choosing $\varepsilon_{1}=0$ instead of $\varepsilon_{1}>0$, we obtain a weaker version of that theorem. The following result is analogous to it.

Lemma 3.5 Let $X$ be a normed linear space and let $x, y \in S_{X}$ with $x \perp_{B}^{\varepsilon} y$ for some $\varepsilon \in[0,1)$. Then there exists $\delta \in(0,1-\varepsilon)$ such that $x \perp_{B}^{\varepsilon+\delta} z$ for all $z \in B(y, \delta) \cap S_{X}$.

Proof. Since $x \perp_{B}^{\varepsilon} y$ for some $\varepsilon \in[0,1)$, there exists $f \in J(x)$ such that $|f(y)| \leq \varepsilon$. Now, if we choose $\delta \in(0,1-\varepsilon)$, then for all $z \in B(y, \delta) \cap S_{X}$, we have, 


$$
|f(z)|=|f(z)-f(y)+f(y)| \leq|f(y)|+|f(z)-f(y)| \leq \varepsilon+\delta .
$$

Thus $x \perp_{B}^{\varepsilon+\delta} z$ for all $z \in B(y, \delta) \cap S_{X}$.

Lemma 3.5 says that the C-approximate orthogonality is stable with respect to the second vector (small perturbation of it does not cause loss of approximate orthogonality). However, as opposed to D-approximate orthogonality (see Theorem 2.1), there is no analogous stability with respect to the first vector. Namely, as it can be observed in the following example, the implication

$$
x \perp_{B}^{\varepsilon} y \quad \Rightarrow \quad \exists \delta \in(0,1-\varepsilon) \forall z \in B(x, \delta) \cap S_{X}: z \perp_{B}^{\varepsilon+\delta} y
$$

need not be true.

Example 3.6 Let $X=\mathbb{R}^{2}$ with the maximum norm. Let $\varepsilon \in[0,1)$ and take $x=(1,1), y=(-1,-\varepsilon), y_{0}=(-1,0)$. Since $x \perp_{B} y_{0}$ and $\left\|y-y_{0}\right\|=\varepsilon$, it follows, via (1.2), that $x \perp_{B}^{\varepsilon} y$. Assuming that (3.1) is true we take a suitable $\delta \in(0,1-\varepsilon)$ and set $z=\left(1,1-\frac{\delta}{2}\right)$. Then $z \in B(x, \delta) \cap S_{X}$ whence $z \perp \varepsilon_{B}^{\prime} y$ with $\varepsilon^{\prime}=\varepsilon+\delta<1$. It would mean, again by (1.2), that there exists $y^{\prime} \in S_{X}$ such that $z \perp_{B} y^{\prime}$ and $\left\|y^{\prime}-y\right\| \leq \varepsilon^{\prime}<1$. However, since $z^{\perp} \cap S_{X}=\{(0,1),(0,-1)\}$, we have $y^{\prime}=(0,1)$ or $y^{\prime}=(0,-1)$ but in both cases $\left\|y-y^{\prime}\right\| \geq 1-$ a contradiction.

We now prove a complete characterization of the C-approximate rightsymmetry on any compact subset of $S_{X}$ for any normed linear space $X$.

Theorem 3.7 Let $X$ be a normed linear space and let $\mathcal{A} \subseteq S_{X}$ be a compact subset. Then any $y \in \mathcal{A}$ is $C$-approximately right-symmetric on $\mathcal{A}$ if and only if the local property $(\mathrm{P})$ on $\mathcal{A}$ holds for each $x \in \mathcal{A}$.

Proof. We first prove the necessary part. Suppose on the contrary that $x \in \mathcal{A}$ is such that $x$ fails to have the local property $(\mathrm{P})$ on $\mathcal{A}$. This implies that there exists $y \in x^{\perp} \cap \mathscr{A}(x) \cap \mathcal{A}$. Now, the C-approximate right-symmetry of $y \in \mathcal{A}$ on $\mathcal{A}$ implies that there exist $\varepsilon \in[0,1)$ and $g \in J(y)$ such that $|g(x)| \leq \varepsilon$. This leads to a contradiction since $y \in \mathscr{A}(x)$ implies $|g(x)|=1$.

We now prove the sufficient part. Suppose on the contrary that there exists $y \in \mathcal{A}$ such that $y$ is not $\mathrm{C}$-approximately right-symmetric on $\mathcal{A}$. Observe that if $z \in^{\perp} y \cap \mathcal{A}$, then it follows from similar arguments as those used in Lemma 3.4 that there exists $\varepsilon_{z, y} \in[0,1)$ such that $y \perp_{B}^{\varepsilon_{z, y}} z$. Let $\varepsilon_{z, y}^{*}$ be the infimum of all such $\varepsilon_{z, y}$. By the assumption $y$ is not C-approximately rightsymmetric on $\mathcal{A}$, this implies that $\varepsilon_{y}=\sup _{z \in \in^{\perp} \cap \mathcal{A}} \varepsilon_{z, y}^{*}=1$. Thus we can find $\left\{z_{n}\right\} \subseteq{ }^{\perp} y \cap \mathcal{A}$ such that $y \perp_{B}^{\varepsilon_{n}^{*}} z_{n}$ for $\varepsilon_{n} \nearrow 1$. Now, from the compactness of $\mathcal{A}$ we can find a convergent subsequence of $\left\{z_{n}\right\}$ which we again denote by $\left\{z_{n}\right\}$. Let $z_{n} \longrightarrow z_{0}$, then by continuity of the norm and compactness of $\mathcal{A}$, it follows that $z_{0} \in^{\perp} y \cap \mathcal{A}$. Again, from similar arguments as those used in Lemma 3.4, it follows that $y \perp_{B}^{\varepsilon_{z_{0}, y}} z_{0}$ for some $\varepsilon_{z_{0}, y} \in[0,1)$. 
Now, if we choose $\delta \in\left(0,1-\varepsilon_{z_{0}, y}\right)$, then it follows from Lemma 3.5 that there exists some $m \in \mathbb{N}$ such that $y \perp_{B}^{\varepsilon_{z_{0}, y}+\delta} z_{k}$ for all $k \geq m$. This leads to a contradiction and thus the result follows.

As an immediate consequence of the above theorem, we obtain the following complete characterization of the C-approximate right-symmetry of elements of $S_{X}$ for any finite-dimensional Banach space.

Corollary 3.8 Let $X$ be a finite-dimensional Banach space. Then any $y \in S_{X}$ is C-approximately right-symmetric if and only if the property $(\mathrm{P})$ holds for $X$.

Now, we present a complete characterization of the C-approximate symmetry of the Birkhoff-James orthogonality in finite-dimensional polyhedral Banach spaces. In the proof of this characterization we will use the following result.

Lemma 3.9 Let $X$ be a finite-dimensional polyhedral Banach space. Then for any sequence $\left\{x_{n}\right\} \subseteq S_{X}$, we can find a sub-sequence $\left\{x_{n_{k}}\right\} \subseteq\left\{x_{n}\right\}$ such that $J\left(x_{n_{i}}\right)=J\left(x_{n_{j}}\right)$ for all $i, j \in \mathbb{N}$.

Proof. If infinitely many elements of $\left\{x_{n}\right\}$ are smooth points of $S_{X}$, then by the fact that there are only finitely many faces in $S_{X}$, we can find a sub-sequence $\left\{x_{n_{k}}\right\} \subseteq\left\{x_{n}\right\} \cap \operatorname{sm} S_{X}$ such that all the elements of the sub-sequence $\left\{x_{n_{k}}\right\}$ lie in the interior of the same facet of $S_{X}$. Thus there exists $f \in S_{X *}$ such that $J\left(x_{n_{k}}\right)=\{f\}$ for all $k \in \mathbb{N}$.

Without loss of generality we now assume that all elements of $\left\{x_{n}\right\}$ are non-smooth points. If there exists $x_{0} \in \operatorname{Ext} B_{X}$ such that $x_{n}=x_{0}$ for infinitely many $n$, then clearly the result follows. If not, then we can choose an edge of $S_{X}$ such that its interior contains infinitely many $x_{n}$, as $S_{X}$ has only finitely many edges. It follows from [13, Theorem 2.1] that every supporting functional of any $z \in S_{X}$ is a convex combination of the supporting functionals of the facets containing $z$. Thus the points lying in the interior of the same edge have identical set of support functionals and hence the result follows.

Theorem 3.10 Let $X$ be a finite-dimensional polyhedral Banach space. Then the following properties are equivalent:

(a) the Birkhoff-James orthogonality is C-approximately symmetric in $X$;

(b) any $y \in S_{X}$ is $C$-approximately left-symmetric;

(c) the property $(\mathrm{P})$ holds for $X$;

(d) the local property $(\mathrm{P})$ holds for all $x \in \operatorname{Ext} B_{X}$.

Proof. Observe that $(a)$ trivially implies $(b)$.

Let us now prove $(b) \Rightarrow(c)$. Suppose on the contrary that there exists $x \in S_{X}$ such that the local property (P) fails for $x$. Thus there exists some $y \in x^{\perp} \cap \mathscr{A}(x)$. Now, $y \in \mathscr{A}(x)$ implies that if $g \in J(y)$, then $|g(x)|=1$. Thus 
there does not exist any $\varepsilon \in[0,1)$ such that $y \perp_{B}^{\varepsilon} x$. This contradicts that $x$ is C-approximately left-symmetric and $(c)$ follows.

$(c) \Rightarrow(d)$ is obvious and $(d) \Rightarrow(c)$ follows from Theorem 3.3.

We now show that $(c) \Rightarrow(b)$. Let $y \in S_{X}$ and $z \in y^{\perp} \cap S_{X}$; then from Lemma 3.4 it follows that $z \perp_{B}^{\varepsilon_{y}, z} y$ for some $\varepsilon_{y, z} \in[0,1)$. Let $\varepsilon_{y, z}^{*}$ be the infimum of all such $\varepsilon_{y, z}$. Let $\varepsilon_{y}=\sup _{z \in y^{\perp} \cap S_{X}} \varepsilon_{y, z}^{*}$, then $\varepsilon_{y} \leq 1$. If $\varepsilon_{y}=1$, then we can find $\left\{z_{n}\right\} \subseteq y^{\perp} \cap S_{X}$ such that $z_{n} \perp_{B}^{\varepsilon_{n}^{*}} y$ for $\varepsilon_{n} \nearrow 1$. Now, from the compactness of $S_{X}$ we can find a convergent sub-sequence of $\left\{z_{n}\right\}$, which we again denote by $\left\{z_{n}\right\}$, and let $z_{n} \longrightarrow z_{0}$. Then by continuity of the norm it follows that $z_{0} \in y^{\perp} \cap S_{X}$. Using Lemma 3.4, it follows that $z_{0} \perp_{B}^{\varepsilon_{y, z_{0}}} y$ for some $\varepsilon_{y, z_{0}} \in[0,1)$. If $z_{n}=z_{0}$ for infinitely many $n$ 's, then clearly we obtain a contradiction. Thus without loss of generality we can assume that $z_{n} \neq z_{0}$ for all $n \in \mathbb{N}$.

Now, it follows from Lemma 3.9 that we can choose a sub-sequence $\left\{z_{n_{k}}\right\} \subseteq\left\{z_{n}\right\}$ such that $J\left(z_{n_{i}}\right)=J\left(z_{n_{j}}\right)$ for all $i, j \in \mathbb{N}$.

Let $\varepsilon_{1} \in[0,1)$ be such that $z_{n_{1}} \perp_{B}^{\varepsilon_{1}} y$. Then the choice of the sub-sequence $\left\{z_{n_{k}}\right\}$ will ensure that $z_{n_{k}} \perp_{B}^{\varepsilon_{1}} y$ for all $k \geq 1$. This leads to a contradiction. Thus $\varepsilon_{y}<1, z \perp_{B}^{\varepsilon_{y}} y$ and hence $y$ is C-approximately left-symmetric.

Now, we show that $(b) \Rightarrow(a)$. Suppose on the contrary that the BirkhoffJames orthogonality is not $\mathrm{C}$-approximately symmetric in $X$. From the equivalence of $(b)$ and $(c)$, it follows that each $x \in S_{X}$ has the local property (P). If $x, y \in S_{X}$ are such that $x \perp_{B} y$, then it follows by Lemma 3.4 that there exists $\varepsilon_{x, y} \in[0,1)$ such that $y \perp_{B}^{\varepsilon_{x, y}} x$. Let $\left\{x_{n}\right\},\left\{y_{n}\right\} \subseteq S_{X}$ be such that $x_{n} \perp_{B} y_{n}$, $y_{n} \perp_{B}^{\varepsilon_{n}^{*}} x_{n}$ for $\varepsilon_{n} \nearrow 1$. Since $S_{X}$ is compact, it follows that there exist convergent sub-sequences of $\left\{x_{n}\right\},\left\{y_{n}\right\}$, which we again denote by $\left\{x_{n}\right\},\left\{y_{n}\right\}$, respectively. Let $x_{0}, y_{0} \in S_{X}$ be such that $x_{n} \longrightarrow x_{0}$ and $y_{n} \longrightarrow y_{0}$. Now, from the continuity of the norm it follows that $x_{0} \perp_{B} y_{0}$. Using Lemma 3.4, we can find $\varepsilon_{x_{0}, y_{0}} \in[0,1)$ such that $y_{0} \perp_{B}^{\varepsilon_{x_{0}}, y_{0}} x_{0}$.

We now prove that we can choose $\left\{x_{n}\right\}$ such that $x_{n} \neq x_{0}$ for almost all $n \in \mathbb{N}$. If there exists a sub-sequence $\left\{x_{n_{k}}\right\} \subseteq\left\{x_{n}\right\}$ such that $x_{n_{k}}=x_{0}$ for all $k \in \mathbb{N}$, then $x_{0} \perp_{B} y_{n_{k}}$ for all $k \in \mathbb{N}$. Then by taking $\mathcal{A}=S_{X}$ in Theorem 3.7, it follows that $y_{n_{k}} \perp_{B}^{\varepsilon_{x_{0}}} \quad x_{0}$ for some $\varepsilon_{x_{0}} \in[0,1)$ and for all $k \in \mathbb{N}$. Thus $y_{n_{k}} \perp_{B}^{\varepsilon_{x_{0}}} x_{n_{k}}$ for some $\varepsilon_{x_{0}} \in[0,1)$ and for all $k \in \mathbb{N}$. Clearly, this contradicts that $y_{n} \perp_{B}^{\varepsilon_{n}^{*}} x_{n}$ for $\varepsilon_{n} \nearrow 1$. Thus we can assume that $x_{n} \neq x_{0}$ for almost all $n \in \mathbb{N}$. Also, by using the similar arguments we can assume that $y_{n} \neq y_{0}$ for almost all $n \in \mathbb{N}$.

It follows from Lemma 3.9 that we can find sub-sequences $\left\{x_{n_{k}}\right\},\left\{y_{n_{k}}\right\}$ of $\left\{x_{n}\right\},\left\{y_{n}\right\}$, respectively, such that $J\left(x_{n_{i}}\right)=J\left(x_{n_{j}}\right)$ and $J\left(y_{n_{i}}\right)=J\left(y_{n_{j}}\right)$ for all $i, j \in \mathbb{N}$. Observe that $x_{n_{k}} \perp_{B} y_{n_{k}}$ for all $k \in \mathbb{N}$ and each $x \in S_{X}$ has local property $(\mathrm{P})$ which implies the following:

(i) if $\left\{x_{n_{k}}\right\},\left\{y_{n_{k}}\right\} \subseteq S_{X} \backslash\left(\operatorname{sm} S_{X} \cup \operatorname{Ext} B_{X}\right)$, then elements of $\left\{x_{n_{k}}\right\}$ and $\left\{y_{n_{k}}\right\}$ lie in the interiors of different edges of $S_{X}$. 
(ii) for all other cases elements of $\left\{x_{n_{k}}\right\},\left\{y_{n_{k}}\right\}$ lie on different facets of $S_{X}$. Now, from the choice of the sub-sequences $\left\{x_{n_{k}}\right\}$ and $\left\{y_{n_{k}}\right\}$ it follows that

$$
x_{n_{i}} \perp_{B} y_{n_{j}} \text { for all } i, j \in \mathbb{N} \text {. }
$$

Thus $x_{0} \perp_{B} y_{n_{j}}$ for all $j \in \mathbb{N}$. Using $(b)$ we can find $\varepsilon_{x_{0}} \in[0,1)$ such that $y_{n_{j}} \perp_{B}^{\varepsilon_{x_{0}}} x_{0}$ for all $j \in \mathbb{N}$.

Let $f \in J\left(y_{n_{1}}\right)$ be such that $\left|f\left(x_{0}\right)\right| \leq \varepsilon_{x_{0}}$. Now, if we choose $\delta \in(0,1-$ $\left.\varepsilon_{x_{0}}\right)$, then for $z \in B\left(x_{0}, \delta\right) \cap S_{X}$, we have,

$$
|f(z)| \leq\left|f(z)-f\left(x_{0}\right)\right|+\left|f\left(x_{0}\right)\right| \leq \delta+\varepsilon_{x_{0}} .
$$

The choice of $\left\{y_{n_{k}}\right\}$ implies that $f \in J\left(y_{n_{j}}\right)$ for all $j \in \mathbb{N}$. Thus we can find $m \in \mathbb{N}$ such that

$$
y_{n_{j}} \perp_{B}^{\varepsilon_{x_{0}}+\delta} x_{n_{j}} \text { for all } j \geq m .
$$

This clearly contradicts that $x_{n} \perp_{B}^{\varepsilon_{n}^{*}} y_{n}$ for $\varepsilon_{n} \nearrow 1$. Thus the BirkhoffJames orthogonality is $\mathrm{C}$-approximately symmetric in $X$.

In view of Theorems 3.7 and 3.10 we would like to propose the following conjecture.

Conjecture 3.11 Let $X$ be a finite-dimensional Banach space. Then the BirkhoffJames orthogonality is C-approximately symmetric in $X$ if and only if the local property $(\mathrm{P})$ holds for each $x \in \operatorname{Ext} B_{X}$.

The above conjecture is not true in general. In [5, Example 3.7], an infinite-dimensional smooth space (hence the local property $(\mathrm{P})$ holds for each $x \in S_{X}$ ), which is not $\mathrm{C}$-approximately symmetric was constructed.

In [5, Theorem 4.2], Chmieliński and Wójcik proved that in finitedimensional smooth Banach spaces, the Birkhoff--James orthogonality is Capproximately symmetric. We now generalize the result by proving that in uniformly smooth Banach spaces, the Birkhoff-James orthogonality is C-approximately symmetric on any compact subset of $S_{X}$.

Theorem 3.12 Let $X$ be a uniformly smooth Banach space and let $\mathcal{A} \subseteq S_{X}$ be a compact subset. Then the Birkhoff-James orthogonality is C-approximately symmetric on $\mathcal{A}$.

Proof. For $x \in S_{X}$ let $J(x)=\left\{f_{x}\right\}$. First we claim that if $\left\{x_{n}\right\} \subseteq S_{X}$ and $x \in S_{X}$ such that $x_{n} \longrightarrow x$, then $f_{x_{n}} \longrightarrow f_{x}$. Suppose on the contrary that $x_{n} \longrightarrow x$ and $f_{x_{n}} \nrightarrow f_{x}$. Then for given $\varepsilon>0$ there exists $\left\{x_{n_{k}}\right\} \subseteq\left\{x_{n}\right\}$ such that $\left\|f_{x_{n_{k}}}-f_{x}\right\|>\varepsilon$ for all $k \in \mathbb{N}$. Clearly, $\varepsilon<2$. Since $X$ is uniformly smooth, $X^{*}$ is uniformly convex. Thus there exists $\delta(\varepsilon)>0$ such that $\left\|f_{x_{n_{k}}}+f_{x}\right\|<$ $2-\delta(\varepsilon)$ for all $k \in \mathbb{N}$. Also, for all $k \in \mathbb{N}$, we have,

$$
\left\|f_{x_{n_{k}}}+f_{x}\right\| \geq f_{x_{n_{k}}}\left(x_{n_{k}}\right)+f_{x}\left(x_{n_{k}}\right)=1+f_{x}\left(x_{n_{k}}\right) .
$$


As $x_{n_{k}} \longrightarrow x$, we get,

$$
2 \leq \lim _{k \longrightarrow \infty}\left\|f_{x_{n_{k}}}+f_{x}\right\| \leq 2-\delta(\varepsilon) .
$$

This leads to a contradiction and thus our claim follows.

It follows from the smoothness of $X$ that any $x \in S_{X}$ has local property (P). If $x, y \in S_{X}$ are such that $x \perp_{B} y$, then by using Lemma 3.4, we can find $\varepsilon_{x, y} \in[0,1)$ such that $y \perp_{B}^{\varepsilon_{x, y}} x$.

Now, we will prove that the Birkhoff-James orthogonality is C-approximately symmetric on any compact subset $\mathcal{A} \subseteq S_{X}$. Suppose on the contrary that the Birkhoff-James orthogonality is not C-approximately symmetric on some compact subset $\mathcal{A} \subseteq S_{X}$. Thus we can find $\left\{x_{n}\right\},\left\{y_{n}\right\} \subseteq \mathcal{A}$ such that $x_{n} \perp_{B} y_{n}$ and $y_{n} \perp_{B}^{\varepsilon_{n}^{*}} x_{n}$ for some $\varepsilon_{n} \nearrow 1$.

From compactness of $\mathcal{A}$ we can find convergent sub-sequences of $\left\{x_{n}\right\}$, $\left\{y_{n}\right\}$ which we again denote by $\left\{x_{n}\right\}$ and $\left\{y_{n}\right\}$, respectively. Let $x_{0}, y_{0} \in \mathcal{A}$ be such that $x_{n} \longrightarrow x_{0}$ and $y_{n} \longrightarrow y_{0}$. Using continuity of the norm it follows that $x_{0} \perp_{B} y_{0}$ and thus there exists $\varepsilon \in[0,1)$ such that $y_{0} \perp_{B}^{\varepsilon} x_{0}$.

Let $\varepsilon_{1} \in(0,1-\varepsilon)$. Then there exists $m_{1} \in \mathbb{N}$ such that $\left\|f_{y_{n}}-f_{y_{0}}\right\| \leq \varepsilon_{1}$ for all $n \geq m_{1}$. Thus for all $n \geq m_{1}$, we have,

$$
\left|f_{y_{n}}\left(x_{0}\right)\right| \leq\left|f_{y_{n}}\left(x_{0}\right)-f_{y_{0}}\left(x_{0}\right)\right|+\left|f_{y_{0}}\left(x_{0}\right)\right| \leq \varepsilon_{1}+\varepsilon
$$

and this implies

$$
y_{n} \perp_{B}^{\varepsilon+\varepsilon_{1}} x_{0} .
$$

Now, if we choose $\delta \in\left(0,1-\varepsilon-\varepsilon_{1}\right)$, then we can find $m_{2} \in \mathbb{N}$ such that $x_{n} \in B\left(x_{0}, \delta\right)$ for all $n \geq m_{2}$.

Let $m=\max \left\{m_{1}, m_{2}\right\}$. Then for all $z \in B\left(x_{0}, \delta\right)$ and for all $n \geq m$, we have,

$$
\left|f_{y_{n}}(z)\right| \leq\left|f_{y_{n}}(z)-f_{y_{n}}\left(x_{0}\right)\right|+\left|f_{y_{n}}\left(x_{0}\right)\right| \leq\left\|z-x_{0}\right\|+\left|f_{y_{n}}\left(x_{0}\right)\right|<\delta+\varepsilon+\varepsilon_{1} .
$$

Thus

$$
y_{n} \perp_{B}^{\delta+\varepsilon+\varepsilon_{1}} x_{n} \text { for all } n \geq m .
$$

This clearly leads to a contradiction and thus the result follows.

For a normed linear space $X$, the following constant was defined in [16]:

$$
\mathcal{R}(X):=\sup \left\{\|x-y\|: \overline{x y} \subset S_{X}\right\} .
$$

Remark 3.13 If $X$ is a finite-dimensional polyhedral Banach space and $\mathcal{R}(X) \leq$ 1 , then the local property $(\mathrm{P})$ holds for each $x \in \operatorname{Ext} B_{X}$. To see this observe that if the local property $(\mathrm{P})$ fails for $x \in \operatorname{Ext} B_{X}$, then there exists $y \in S_{X}$ such that $x \perp_{B} y$ and if $f \in J(y)$, then either $f \in J(x)$ or $-f \in J(x)$. This implies that one of the following holds true:

(i) $y$ lies in the interior of one of the associated edges of $x$ or $-x$;

(ii) $y$ lies in the interior of one of the associated facets of $x$ or $-x$. 
Thus either $\overline{x y} \subset S_{X}$ or $\overline{-x y} \subset S_{X}$. If $\overline{x y} \subset S_{X}$, then $x \perp_{B} y$ implies

$$
\mathcal{R}(X)>\|x-y\| \geq\|x\|=1 .
$$

Now, we assume that $\overline{-x y} \subset S_{X}$. By the homogeneity of orthogonality and $x \perp_{B} y$ we get $-x \perp_{B} y$. Thus,

$$
\mathcal{R}(X)>\|(-x)-y\| \geq\|-x\|=1 \text {. }
$$

The following example shows that the converse is not true, that is, a two-dimensional polyhedral Banach space satisfying the local property $(\mathrm{P})$ for each $x \in \operatorname{Ext} B_{X}$ need not necessarily satisfy $\mathcal{R}(X) \leq 1$. Consider a twodimensional polyhedral Banach space $X=\mathbb{R}^{2}$, whose unit sphere is determined by the extreme points $v_{1}=(2,2), v_{2}=(1,3), v_{3}=(0,3.5), v_{4}=(-1,3)$, $v_{5}=(-2,2), v_{6}=-v_{1}, v_{7}=-v_{2}, v_{8}=-v_{3}, v_{9}=-v_{4}, v_{10}=-v_{5}$. For this space $\mathcal{R}(X)>1$, Figs. 3 and 4 , show that the local property (P) holds for each $x \in \operatorname{Ext} B_{X}$. In Fig. 3, $f$ is the supporting functional corresponding to the edge $\overline{v_{1} v_{10}}, g$ is the supporting functional corresponding to the edge $\overline{v_{1} v_{2}}$ and $h$ is the supporting functional corresponding to the edge $\overline{v_{2} v_{3}}$. In Fig. $4, h$ is the supporting functional corresponding to the edge $\overline{v_{3} v_{4}}, g$ is the supporting functional corresponding to the edge $\overline{v_{4} v_{5}}$ and $f$ is the supporting functional corresponding to the edge $\overline{v_{5} v_{6}}$.

As a consequence of Theorem 3.10 and Remark 3.13 we obtain the next result.

Theorem 3.14 Let $X$ be a finite-dimensional polyhedral Banach space such that $\mathcal{R}(X) \leq 1$. Then the Birkhoff-James orthogonality is $C$-approximately symmetric.

Remark 3.15. The previous example shows that the converse of the above theorem is not true. It follows also from [5, Corollary 3.9] that for any finitedimensional Banach space $X$ (not necessarily polyhedral) if $\mathcal{R}(X)<1$, then the Birkhoff-James orthogonality is C-approximately symmetric. However, polyhedralness is essential for $\mathcal{R}(X)=1$. Indeed, consider the $l_{2}-l_{\infty}$ norm on the plane (see Fig. 5) and vectors $x=(1,1), y=(0,1)$. Then $x \perp_{B} y$ but $y \not \perp_{B}^{\varepsilon} x$ for any $\varepsilon \in[0,1)$.

\section{C-approximate Symmetry for Two-Dimensional Polyhedral Banach Spaces}

We introduce yet another property of a normed linear space $X$ : if $\overline{x y} \subset S_{X}$ and $\quad x \perp_{B} y, \quad$ then $x, y \in \operatorname{Ext} B_{X}$.

We first prove that in any finite-dimensional polyhedral Banach space property (P1) always implies the local property (P) for each $x \in S_{X}$. 


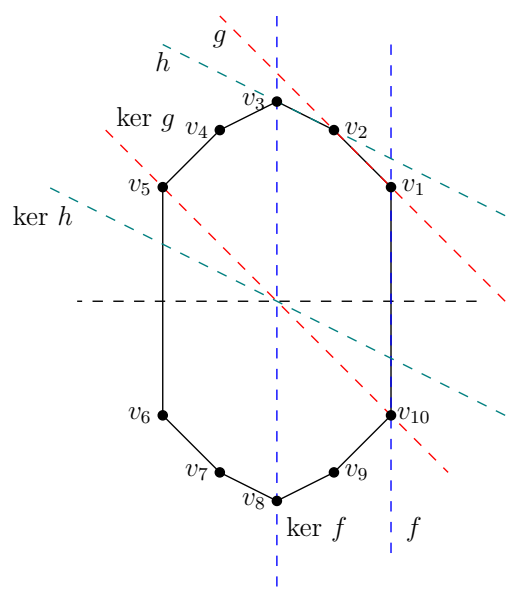

Figure 3. Property $(\mathrm{P})$ and $\mathcal{R}(X)>1$.

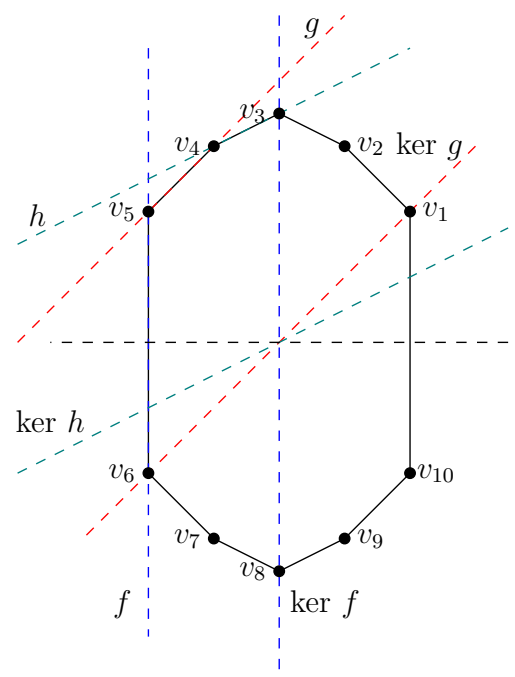

Figure 4. Property $(\mathrm{P})$ and $\mathcal{R}(X)>1$.

Lemma 4.1 Let $X$ be a finite-dimensional polyhedral Banach space such that property $(\mathrm{P} 1)$ holds for $X$. Then the local property $(\mathrm{P})$ holds for each $x \in S_{X}$.

Proof. It follows from Theorem 3.3 that it is sufficient to prove that the local property $(\mathrm{P})$ holds for each $x \in \operatorname{Ext} B_{X}$. Suppose on the contrary that there exists $x \in \operatorname{Ext} B_{X}$ such that the local property (P) fails for $x$. Thus there exists $y \in x^{\perp} \cap S_{X}$ such that if $f \in J(y)$, then either $f \in J(x)$ or $-f \in J(x)$. This clearly shows that either $\overline{x y} \subset S_{X}$ or $\overline{-x y} \subset S_{X}$. Now, property (P1) of 


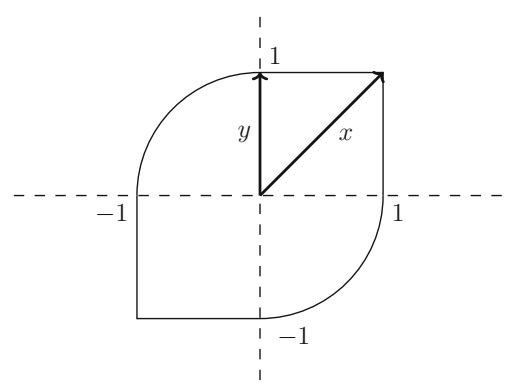

Figure 5. Nonpolyhedral, not approximately symmetric $\ell_{2}-\ell_{\infty}$ space with $\mathcal{R}(X)=1$.

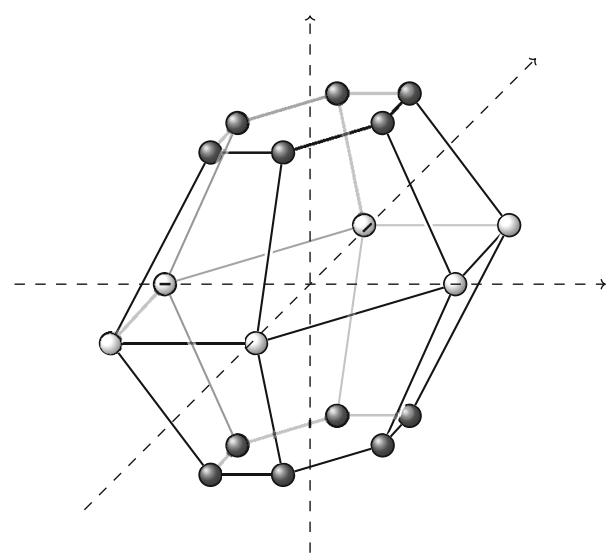

Figure 6. 3-dimensional space with property $(\mathrm{P})$ but not property (P1).

$X$ shows that $y \in \operatorname{Ext} B_{X}$. This contradicts that the local property (P) fails for $x$ and thus the result follows.

We will see that the converse is also true in two-dimensional spaces but in general it is not true. To exhibit this we now give an example of a threedimensional polyhedral Banach space $X$, for which the local property $(\mathrm{P})$ holds for all elements of $S_{X}$ but $X$ fails to have the property (P1).

Example 4.2 Consider a three-dimensional polyhedral Banach space $X=\mathbb{R}^{3}$, whose unit sphere is given in Fig. 6. Observe that the local property (P) holds for each $x \in S_{X}$. If we consider any extreme point of $B_{X}$ which is black in color, then it is orthogonal to all the elements which lie in the intersection of $S_{X}$ and the plane passing through all the extreme points of $B_{X}$ which are white in color. This shows that $X$ fails to have property (P1). 
We now show that for any two-dimensional polyhedral Banach spaces the property (P1) and the local property (P) for each $x \in S_{X}$ are equivalent. The next result provides a complete characterization of the $\mathrm{C}$-approximate symmetry of the Birkhoff-James orthogonality in a two-dimensional polyhedral Banach space.

Theorem 4.3 Let $X$ be a two-dimensional polyhedral Banach space. Then the following properties are equivalent:

(a) the property $(\mathrm{P} 1)$ holds for $X$;

(b) if $x \in \operatorname{Ext} B_{X}$ and $y \in S_{X}$ are such that $x \perp_{B} y$, then $y$ does not lie in the interior of any of the associated edges of $\pm x$;

(c) the local property $(\mathrm{P})$ holds for each $x \in S_{X}$;

(d) the Birkhoff-James orthogonality is C-approximately symmetric in $X$.

Proof. Equivalence of $(c)$ and $(d)$ follows from Theorem 3.10. To complete the proof we now prove the equivalence of $(a),(b)$ and $(c)$.

We first prove $(a) \Rightarrow(b)$. Let $x \in \operatorname{Ext} B_{X}$ and $y \in x^{\perp} \cap S_{X}$. If $y$ does not lie on the associated edges of $\pm x$, then the result follows trivially. Suppose that $y$ lies on one of the associated edges of $x$ or $-x$. Then either $\overline{x y} \subseteq S_{X}$ or $\overline{-x y} \subseteq S_{X}$. Now, it follows from $(a)$ that $y \in \operatorname{Ext} B_{X}$ and thus $(b)$ follows.

We now show $(b) \Rightarrow(c)$. Observe that to show $(c)$, it is sufficient to show that the local property $(\mathrm{P})$ holds for any $x \in \operatorname{Ext} B_{X}$. Let $x \in \operatorname{Ext} B_{X}$ and let $y \in x^{\perp} \cap S_{X}$. It follows from $(b)$ that $y$ does not lie in the interior of any of the associated edges of $\pm x$. Thus there exists some $f \in J(y)$ such that $\pm f \notin J(x)$ and hence $(c)$ follows.

Now, we prove that $(c) \Rightarrow(a)$. Let $x \in S_{X}$ and let $y \in x^{\perp} \cap S_{X}$ be such that $\overline{x y} \subseteq S_{X}$ which implies that $x \in \operatorname{Ext} B_{X}$. The local property $(\mathrm{P})$ of $x$ implies that $y$ cannot be an interior point of any of the associated edges of $x$. Thus $y \in \operatorname{Ext} B_{X}$ and $(a)$ follows. This completes the proof.

Applying Theorem 3.14, we now prove that in any two-dimensional regular polyhedral Banach space with at least 6 vertices, the Birkhoff-James orthogonality is $\mathrm{C}$-approximately symmetric. Regularity here means that all edges of the unit sphere are of the same length with respect to the Euclidean metric and all the interior angles are of the same measure.

Theorem 4.4 Let $X$ be a two-dimensional regular polyhedral space with $2 n$ vertices, $n \in \mathbb{N}, n \geq 3$. Then the Birkhoff-James orthogonality is $C$-approximately symmetric in $X$.

Proof. Without loss of generality we assume that all the vertices of the polygon lie on the Euclidean unit sphere and they are:

$$
v_{j}=\left(\cos \frac{(2 j-1) \pi}{2 n}, \sin \frac{(2 j-1) \pi}{2 n}\right), \quad j=1, \ldots, 2 n
$$

(for $n=3$ and $n=4$ the situation is illustrated in Figs. 7 and 8). 


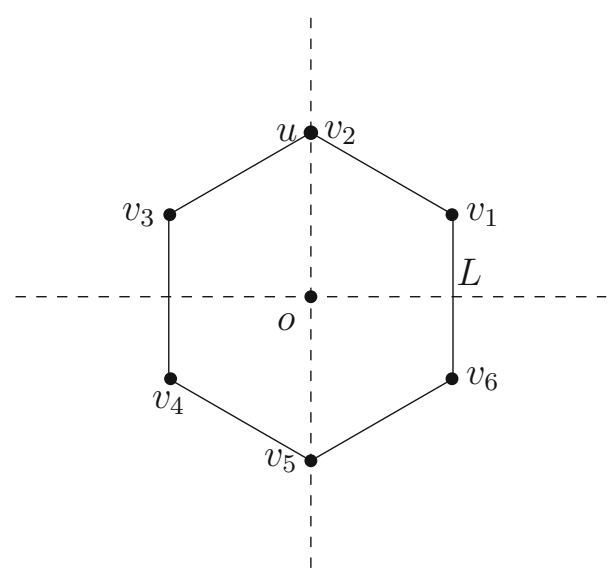

Figure 7. Two-dimensional regular polyhedral space with 6 vertices.

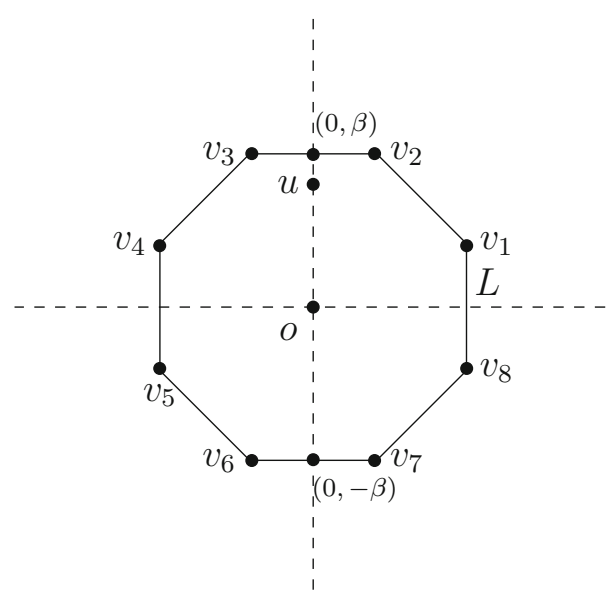

FiguRE 8. Two-dimensional regular polyhedral space with 8 vertices.

Let the ordinate meet the boundary of the polygon at $\pm(0, \beta)$. Then $\beta=1$ if $n$ is odd and $\beta=\cos \frac{\pi}{2 n} \geq \cos \frac{\pi}{8}>0.9$ if $n$ is even.

Let $L=\overline{v_{1} v_{2 n}}$. The value of $\mathcal{R}(X)$ is equal to the length of $L$. To determine the latter one we will translate $L$ by the vector $-v_{2 n}$, which means that $v_{2 n}$ is moved to the origin $o$ and $v_{1}$ to some point $u$ on the ordinate.

The Euclidean length of $L$ is equal to $|L|=2 \sin \frac{\pi}{2 n}$. Then $|L|=1$ for $n=3$ and $|L| \leq 2 \sin \frac{\pi}{8}<0.8<\beta$ for $n \geq 4$. Therefore $u$ lies on the boundary of the polygon for $n=3$ and inside it for $n \geq 4$, hence the length of $L$ (with 


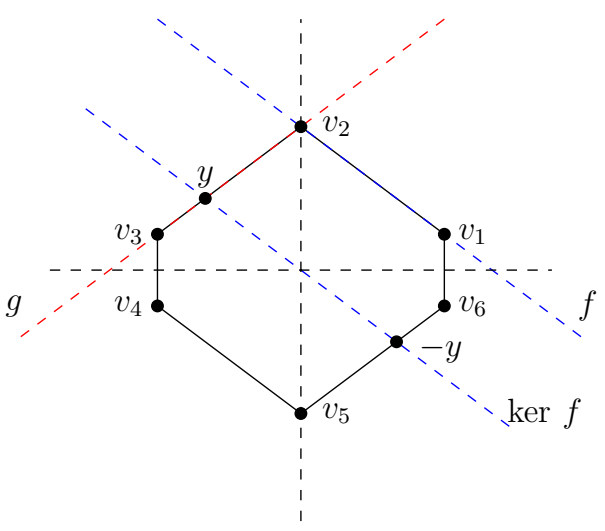

Figure 9. Two-dimensional not regular polyhedral space.

respect to the introduced norm) is not greater than 1 . Thus we have $\mathcal{R}(X) \leq 1$ and the assertion follows now from Theorem 3.14.

The following example shows that in Theorem 4.4, the regularity condition cannot be avoided. Consider a two-dimensional polyhedral Banach space $X=\mathbb{R}^{2}$, whose unit sphere is given in Fig. 9 .

Clearly, $y \in v_{2}^{\perp} \cap S_{X}$. Also, $y$ lies in the interior of the edge $\overline{v_{2} v_{3}}$. The only supporting linear functional for $y$ is the supporting functional $g \in S_{X^{*}}$ corresponding to the edge $\overline{v_{2} v_{3}}$ such that $g(x)=1$ for all $x \in \overline{v_{2} v_{3}}$. Thus $v_{2}$ is not C-approximately left-symmetric.

\section{Acknowledgements}

A joint work on this article began in January 2020, during the first author's visit to the Jadavpur University in Kolkata. The hospitality of Professor Kallol Paul is acknowledged with gratitude. Dr. Debmalya Sain feels elated to acknowledge the motivating presence of his beloved brother Krittish Roy in his life.

\section{Declarations}

Funding The research of Dr. Divya Khurana and Dr. Debmalya Sain is sponsored by Dr. D. S. Kothari Postdoctoral Fellowship under the mentorship of Professor Gadadhar Misra.

Conflicts of interest The authors declare that they have no conflict of interest.

Availability of data and material Not applicable.

Code availability Not applicable. 


\section{References}

[1] Baker, J.M.: A note on compact operators which attain their norm. Pac. J. Math. 82(2), 319-321 (1979)

[2] Birkhoff, G.: Orthogonality in linear metric spaces. Duke Math. J. 1, 169-172 (1935)

[3] Chmieliński, J.: On an $\epsilon$-Birkhoff orthogonality. J. Inequal. Pure Appl. Math. 6(3) (2005) Article 79

[4] Chmieliński, J., Stypuła, T., Wójcik, P.: Approximate orthogonality in normed spaces and its applications. Linear Algebra Appl. 531, 305-317 (2017)

[5] Chmieliński, J., Wójcik, P.: Approximate symmetry of Birkhoff orthogonality. J. Math. Anal. Appl. 461, 625-640 (2018)

[6] Dragomir, S.S.: On approximation of continuous linear functionals on normed linear spaces. An. Univ. Timişoara Ser. Ştiinţ. Mat. 29(1), 51-58 (1991)

[7] James, R.C.: Orthogonality and linear functionals in normed linear spaces. Trans. Am. Math. Soc. 61, 265-292 (1947)

[8] James, R.C.: Inner product in normed linear spaces. Bull. Am. Math. Soc. 53, 559-566 (1947)

[9] Mal, A., Sain, D., Paul, K.: On some geometric properties of operator spaces. Banach J. Math. Anal. 13(1), 174-191 (2019)

[10] Megginson, R.E.: An Introduction to Banach Space Theory. Graduate Texts in Mathematics, vol. 183. Springer, New York (1998). xx+596 pp

[11] Paul, K., Sain, D., Mal, A.: Approximate Birkhoff-James orthogonality in the space of bounded linear operators. Linear Algebra Appl. 537, 348-357 (2018)

[12] Sain, D.: Birkhoff-James orthogonality of linear operators on finite dimensional Banach spaces. J. Math. Anal. Appl. 447(2), 860-866 (2017)

[13] Sain, D., Paul, K., Bhunia, P., Bag, S.: On the numerical index of polyhedral Banach spaces. Linear Algebra Appl. 577, 121-133 (2019)

[14] Sain, D., Paul, K., Mal, A.: On approximate Birkhoff-James orthogonality and normal cones in a normed space. J. Convex Anal. 26(1), 341-351 (2019)

[15] Sen, J., Mal, A., Paul, K.: Characterization of approximate Birkhoff-James orthogonality sets in a Banach space. J. Convex Anal. 27(3), 881-892 (2020)

[16] Stypuła, T., Wójcik, P.: Characterizations of rotundity and smoothness by approximate orthogonalities. Ann. Math. Sil. 30, 183-201 (2016)

Jacek Chmieliński

Department of Mathematics

Pedagogical University of Krakow

Podchorążych 2

30-084 Kraków

Poland

e-mail: jacek.chmielinski@up.krakow.pl 
Divya Khurana and Debmalya Sain

Department of Mathematics

Indian Institute of Science, Bengaluru

Bengaluru Karnataka 560012

India

e-mail: divyakhurana11@gmail.com;

saindebmalya@gmail.com

Received: January 22, 2021.

Accepted: May 17, 2021.

Publisher's Note Springer Nature remains neutral with regard to jurisdictional claims in published maps and institutional affiliations. 